\title{
Incorporation of Polymer-Grafted Cellulose Nanocrystals into Latex-Based Pressure Sensitive Adhesives
}

Michael V. Kiriakou, ${ }^{1}$ Amir Saeid Pakdel, ${ }^{2}$ Richard M. Berry, ${ }^{3}$ Todd Hoare, ${ }^{1}$ Marc A. Dubé, ${ }^{2}$

$$
\text { Emily D. Cranston }{ }^{1,4,5, *}
$$

${ }^{1}$ Department of Chemical Engineering, McMaster University, 1280 Main Street West, ON, L8S 4L7, Canada

${ }^{2}$ Department of Chemical and Biological Engineering, Center for Catalysis Research and Innovation, University of Ottawa, 161 Louis Pasteur Pvt., Ottawa, ON, K1N 6N5, Canada

${ }^{3}$ CelluForce Inc., 625 President-Kennedy Avenue, Montreal, QC, H3A 1K2, Canada

${ }^{4}$ Departments of Wood Science, University of British Columbia, 2424 Main Mall, Vancouver, BC, V6T 1Z4, Canada

${ }^{5}$ Department of Chemical and Biological Engineering, University of British Columbia, 2360 East Mall, Vancouver, BC, V6T 1Z3, Canada

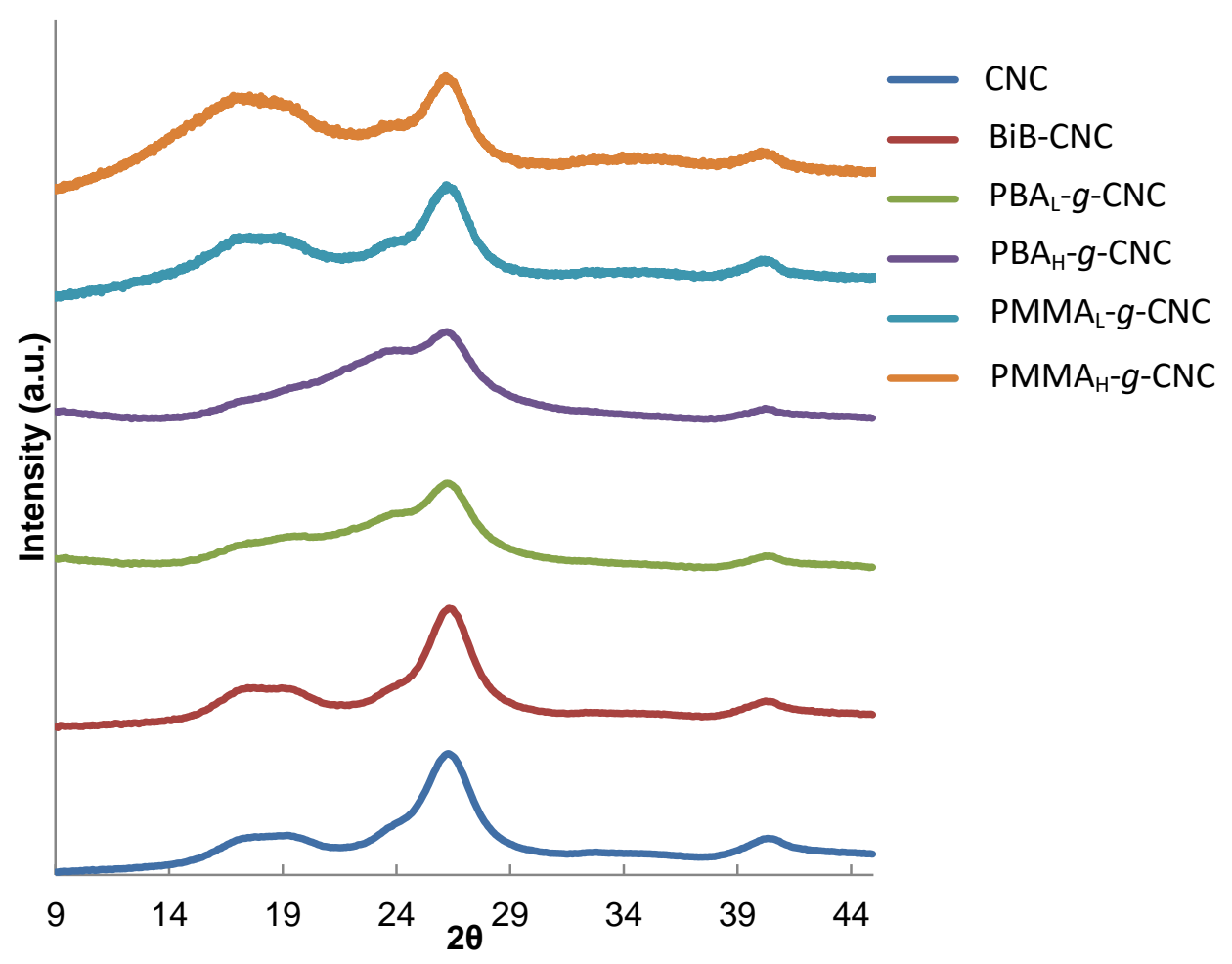

Figure S1. XRD spectra for $\mathrm{CNC}, \mathrm{BiB}-\mathrm{CNC}$ and $g-\mathrm{CNC}$ samples. 


\section{Supporting Information}

Table S1. Percentage of total added mass of CNCs recovered from the BA monomer phase during the water-BA monomer partitioning experiment.

\begin{tabular}{ll}
\hline Component & \% Mass in Monomer (BA) Phase \\
\hline CNC & $<1$ \\
PBA $_{L}-g-C N C$ & 75 \\
PBA $_{H}-$ - $-\mathrm{CNC}$ & 92 \\
PMMA $_{L}-$-CNC & 63 \\
PMMA $_{H}-g-C N C$ & 40
\end{tabular}

\section{Statistical analysis of PSA testing}

To evaluate statistically significant differences between PSA testing results, ANOVA was applied to each sample set. A significance level of $95 \%$ was used, and the resulting $p$-values were compared to assess the null hypothesis (i.e., all population means are equal) as shown in Tables S2-S4.

Table S2. ANOVA charts for PSA tack analysis.

Analysis of Variance
\begin{tabular}{lrrrrr} 
Source & DF & Adj SS & Adj MS & F-Value & P-Value \\
\hline Factor & 12 & 198900 & 16575.0 & 53.54 & 0.000 \\
Error & 65 & 20121 & 309.6 & & \\
Total & 77 & 219022 & & &
\end{tabular}

\section{Model Summary

\begin{tabular}{rrrr} 
S & R-sq & R-sq(adj) & R-sq(pred) \\
\hline $17.594390 .81 \%$ & $89.12 \%$ & $86.77 \%$
\end{tabular}




\begin{tabular}{|c|c|c|c|c|}
\hline Factor & $\mathbf{N}$ & Mean & StDev & $95 \% \mathrm{Cl}$ \\
\hline No CNCs & 6 & 96.12 & 8.78 & $(81.77,110.46)$ \\
\hline 0.5 phm CNC-W & 6 & 71.53 & 15.30 & $(57.19,85.88)$ \\
\hline 0.5 phm CNC-M & 6 & 53.32 & 13.26 & $(38.97,67.66)$ \\
\hline $0.5 \mathrm{phm} \mathrm{PBA}_{\mathrm{L}-\mathrm{g}-\mathrm{CNC}}$ & 6 & 175.57 & 19.17 & $(161.22,189.92)$ \\
\hline $0.5 \mathrm{phm} \mathrm{PBA}_{\mathrm{H}^{-} \mathrm{g}-\mathrm{CNC}}$ & 6 & 128.07 & 16.95 & $(113.72,142.41)$ \\
\hline 0.5 phm PMMA A $-g-C N C$ & 6 & 247.13 & 21.09 & $(232.78,261.47)$ \\
\hline $0.5 \mathrm{phm} \mathrm{PMMA}_{\mathrm{H}}-\mathrm{g}-\mathrm{CNC}$ & 6 & 155.54 & 18.55 & $(141.20,169.89)$ \\
\hline 1 phm CNC-W & 6 & 166.72 & 11.57 & $(152.37,181.07)$ \\
\hline 1 phm CNC-M & 6 & 93.50 & 31.70 & $(79.10,107.80)$ \\
\hline $1 \mathrm{phm} P B A_{L}-g-C N C$ & 6 & 187.64 & 14.69 & $(173.29,201.98)$ \\
\hline $1 \mathrm{phm} \mathrm{PBA}_{\mathrm{H}}-\mathrm{g}-\mathrm{CNC}$ & 6 & 136.13 & 14.48 & $(121.78,150.47)$ \\
\hline 1 phm $P M M A_{L}-g-C N C$ & 6 & 166.12 & 10.89 & $(151.77,180.47)$ \\
\hline 1 phm PMMA $_{H-g}-\mathrm{CNC}$ & 6 & 132.22 & 20.28 & $(117.88,146.57)$ \\
\hline
\end{tabular}

Table S3. ANOVA chart for PSA peel strength analysis.

Analysis of Variance
\begin{tabular}{|rrrrrr} 
Source & DF & Adj SS & Adj MS & F-Value & P-Value \\
\hline Factor & 12 & 15986.2 & 1332.19 & 116.14 & 0.000 \\
Error & 65 & 745.6 & 11.47 & & \\
Total & 77 & 16731.9 & & &
\end{tabular}

\section{Model Summary

\begin{tabular}{rrrr} 
S & R-sq & R-sq(adj) & R-sq(pred) \\
\hline $3.3868795 .54 \%$ & $94.72 \%$ & $93.58 \%$
\end{tabular}




\begin{tabular}{|c|c|c|c|c|}
\hline Factor & $\mathbf{N}$ & Mean & StDev & $95 \% \mathrm{Cl}$ \\
\hline No CNCs & 6 & 6.92 & 0.72 & $(4.16,9.68)$ \\
\hline 0.5 phm CNC-W & 6 & 6.60 & 1.04 & $(3.84,9.36)$ \\
\hline 0.5 phm CNC-M & 6 & 8.80 & 0.54 & $(6.04,11.56)$ \\
\hline $0.5 \mathrm{phm}$ PBA $-\mathrm{g}-\mathrm{CNC}$ & 6 & 19.09 & 3.75 & $(16.33,21.85)$ \\
\hline $0.5 \mathrm{phm} \mathrm{PBA}_{H}-\mathrm{g}-\mathrm{CNC}$ & 6 & 15.69 & 2.47 & $(12.93,18.45)$ \\
\hline $0.5 \mathrm{phm} P M M A_{L}-\mathrm{g}-\mathrm{CNC}$ & 6 & 60.35 & 10.79 & $(57.59,63.12)$ \\
\hline $0.5 \mathrm{phm} \mathrm{PMMA}_{\mathrm{H}}-\mathrm{g}-\mathrm{CNC}$ & 6 & 14.46 & 2.39 & $(11.69,17.22)$ \\
\hline 1 phm CNC-W & 6 & 6.21 & 0.30 & $(3.45,8.97)$ \\
\hline 1 phm CNC-M & 6 & 4.01 & 0.87 & $(1.25,6.78)$ \\
\hline $1 \mathrm{phm} P B A_{L}-g-C N C$ & 6 & 7.78 & 0.90 & $(5.01,10.54)$ \\
\hline $1 \mathrm{phm} \mathrm{PBA}_{\mathrm{H}-\mathrm{g}-\mathrm{CNC}}$ & 6 & 5.08 & 0.76 & $(2.32,7.85)$ \\
\hline 1 phm PMMAL-g-CNC & 6 & 11.56 & 1.46 & $(8.80,14.32)$ \\
\hline 1 phm РMMA ${ }_{H}-\mathrm{g}-\mathrm{CNC}$ & 6 & 5.15 & 0.70 & $(2.39,7.92)$ \\
\hline
\end{tabular}

Table S4. ANOVA chart for PSA shear strength analysis.

Analysis of Variance
\begin{tabular}{lrrrrr} 
Source & DF & Adj SS & Adj MS & F-Value & P-Value \\
\hline Factor & 12 & 13441.1 & 1120.09 & 89.49 & 0.000 \\
Error & 65 & 813.6 & 12.52 & & \\
Total & 77 & 14254.7 & & &
\end{tabular}

\section{Model Summary}

\begin{tabular}{rrrr} 
S & R-sq & R-sq(adj) & R-sq(pred) \\
\hline $3.5378294 .29 \%$ & $93.24 \%$ & $91.78 \%$
\end{tabular}




\section{Supporting Information}

\section{Means}

\begin{tabular}{|c|c|c|c|c|}
\hline Factor & $\mathbf{N}$ & Mean & StDev & $95 \% \mathrm{Cl}$ \\
\hline No CNCs & 6 & 4.51 & 1.73 & $(1.62,7.39)$ \\
\hline 0.5 phm CNC-W & 6 & 37.64 & 0.01 & $(34.76,40.52)$ \\
\hline 0.5 phm CNC-M & 6 & 0.77 & 0.28 & $(-2.11,3.66)$ \\
\hline $0.5 \mathrm{phm}$ PBA $-\mathrm{g}-\mathrm{CNC}$ & 6 & 8.22 & 2.25 & $(5.34,11.11)$ \\
\hline $0.5 \mathrm{phm} \mathrm{PBA}-\mathrm{g}-\mathrm{CNC}$ & 6 & 5.43 & 0.73 & $(2.54,8.31)$ \\
\hline $0.5 \mathrm{phm}$ PMMA $\mathrm{L}_{\mathrm{L}} \mathrm{g}-\mathrm{CNC}$ & 6 & 4.57 & 0.63 & $(1.68,7.45)$ \\
\hline $0.5 \mathrm{phm} \mathrm{PMMA}_{\mathrm{H}^{-}-\mathrm{g}-\mathrm{CNC}}$ & 6 & 7.55 & 1.34 & $(4.67,10.44)$ \\
\hline 1 phm CNC-W & 6 & 40.86 & 3.64 & $(37.97,43.74)$ \\
\hline 1 phm CNC-M & 6 & 34.69 & 8.30 & $(31.81,37.58)$ \\
\hline $1 \mathrm{phm} P B A_{L}-\mathrm{g}-\mathrm{CNC}$ & 6 & 20.43 & 3.10 & $(17.55,23.31)$ \\
\hline 1 phm $\mathrm{PBA}_{H}-\mathrm{g}-\mathrm{CNC}$ & 6 & 15.29 & 5.93 & $(12.40,18.17)$ \\
\hline 1 phm PMMA L-g-CNC & 6 & 10.49 & 1.19 & $(7.61,13.38)$ \\
\hline $1 \mathrm{phm} \mathrm{PMMA}_{H}-\mathrm{g}-\mathrm{CNC}$ & 6 & 11.69 & 4.86 & $(8.80,14.57)$ \\
\hline
\end{tabular}

Pooled StDev $=3.53782$

Based on the results of the ANOVA analysis, the null hypothesis was rejected in all cases (i.e., the p-value $<$ significance value). From this, post-hoc analysis was performed to determine which means were statistically different for each ANOVA analysis. Tukey tests were then used to compare all possible pairs of means based on the studentized range distribution $(q)$. The test is as follows:

$$
\begin{gathered}
q_{c r i t}>q_{\alpha} \\
q_{c r i t}=\frac{\bar{x}_{\text {max }}-\bar{x}_{\text {min }}}{\sqrt{\frac{M S_{W}}{n}}}
\end{gathered}
$$

where $q_{\alpha}$ is taken for the studentized range $q$ table for a given number of groups and degrees of freedom, $n$ is the number of samples within a group, and $M S_{W}$ is the pooled variance. In the case where the above condition is true (i.e., $q_{c r i t}$ larger than the $q_{\alpha}$ value from the studentized range distribution), then a significant difference between the two compared groups is present. Tables S5-S7 summarize the findings of a post-hoc Tukey test for all ANOVA groups. 


\section{Supporting Information}

Table S5. Tukey pairwise comparison summary for PSA tack testing. Means that do not share a letter are significantly different.

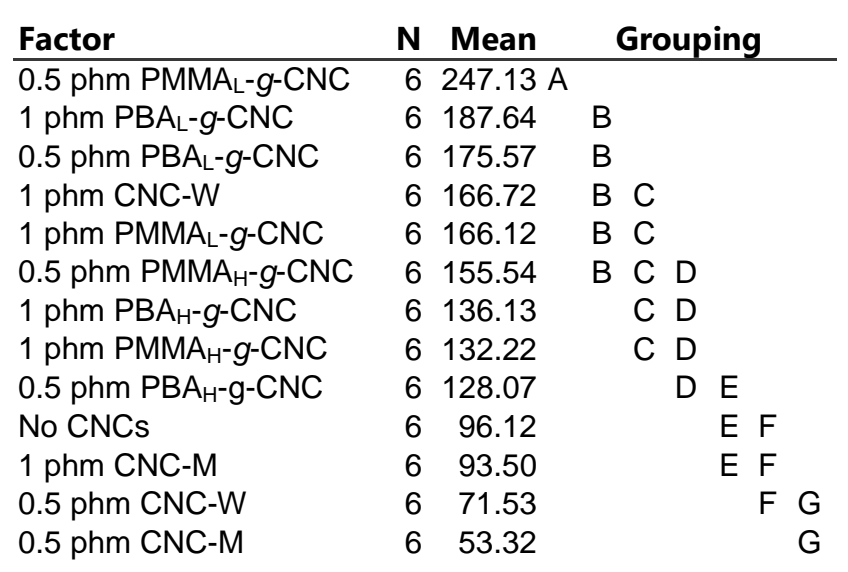

Table S6. Tukey pairwise comparison summary for PSA peel strength testing. Means that do not share a letter are significantly different.

\begin{tabular}{|c|c|c|c|c|c|c|}
\hline Factor & $\mathbf{N}$ & Mean & \multicolumn{4}{|c|}{ Grouping } \\
\hline $0.5 \mathrm{phm} \mathrm{PMMA}_{\mathrm{L}-\mathrm{g}-\mathrm{CNC}}$ & 6 & $60.35 \mathrm{~A}$ & & & & \\
\hline $0.5 \mathrm{phm} \mathrm{PBA}_{\mathrm{L}-g-\mathrm{CNC}}$ & 6 & 19.09 & B & & & \\
\hline $0.5 \mathrm{phm} \mathrm{PBA}_{\mathrm{H}-\mathrm{g}-\mathrm{CNC}}$ & 6 & 15.69 & B & C & & \\
\hline $0.5 \mathrm{phm} \mathrm{PMMA}_{\mathrm{H}}-\mathrm{g}-\mathrm{CNC}$ & 6 & 14.46 & B & C & D & \\
\hline $1 \mathrm{phm} P M M A_{L}-g-\mathrm{CNC}$ & 6 & 11.56 & & C & D & $E$ \\
\hline 0.5 phm CNC-M & 6 & 8.80 & & & D & E F \\
\hline No CNCs & 6 & 7.78 & & & D & \\
\hline 0.5 phm CNC-W & 6 & 6.92 & & & & \\
\hline $1 \mathrm{phm}$ CNC-W & 6 & 6.60 & & & & \\
\hline No CNCs & 6 & 6.21 & & & & \\
\hline 1 phm $\mathrm{PMMA}_{\mathrm{H}}-g-\mathrm{CNC}$ & 6 & 5.15 & & & & \\
\hline $1 \mathrm{phm} \mathrm{PBA}_{\mathrm{H}}-\mathrm{g}-\mathrm{CNC}$ & 6 & 5.08 & & & & $\mathrm{E}$ \\
\hline $1 \mathrm{phm}$ CNC-M & 6 & 4.01 & & & & \\
\hline
\end{tabular}


Table S7. Tukey pairwise comparison summary for PSA shear strength testing. Means that do not share a letter are significantly different.

\begin{tabular}{|c|c|c|c|c|c|c|}
\hline Factor & $\mathbf{N}$ & Mean & \multicolumn{4}{|c|}{ Grouping } \\
\hline $1 \mathrm{phm}$ CNC-W & 6 & $40.86 \mathrm{~A}$ & & & & \\
\hline $0.5 \mathrm{phm}$ CNC-W & 6 & $37.64 \mathrm{~A}$ & & & & \\
\hline 1 phm CNC-M & 6 & $34.69 \mathrm{~A}$ & & & & \\
\hline 1 phm PBA $A_{L}-g-C N C$ & 6 & 20.43 & B & & & \\
\hline $1 \mathrm{phm} \mathrm{PBA}_{\mathrm{H}-\mathrm{g}-\mathrm{CNC}}$ & 6 & 15.29 & B & C & & \\
\hline $1 \mathrm{phm} \mathrm{PMMA_{H } - g - C N C}$ & 6 & 11.69 & & & D & \\
\hline 1 phm $P_{M M A_{L}-g-C N C}$ & 6 & 10.49 & & C & & $E$ \\
\hline $1 \mathrm{phm} \mathrm{PBA}_{\mathrm{L}}-g-\mathrm{CNC}$ & 6 & 8.22 & & & D & $\mathrm{E}$ \\
\hline 0.5 phm $\mathrm{PMMA}_{\mathrm{H}}-\mathrm{g}-\mathrm{CNC}$ & 6 & 7.55 & & & $\mathrm{D}$ & E F \\
\hline $0.5 \mathrm{phm} \mathrm{PBA}_{\mathrm{H}-\mathrm{g}-\mathrm{CNC}}$ & 6 & 5.43 & & & D & \\
\hline $0.5 \mathrm{phm}$ PMMA $A_{L}-g-C N C$ & 6 & 4.57 & & & & \\
\hline No CNCs & 6 & 4.51 & & & & \\
\hline 0.5 phm CNC-M & 6 & 0.77 & & & & $\mathrm{~F}$ \\
\hline
\end{tabular}

